\title{
An empirical method to correct for temperature-dependent variations in the overlap function of $\mathrm{CHM15k}$ ceilometers
}

\author{
Maxime Hervo $^{1}$, Yann Poltera ${ }^{1, a}$, and Alexander Haefele ${ }^{1}$ \\ ${ }^{1}$ MeteoSwiss, Payerne, Switzerland \\ ${ }^{a}$ now at: Institute for Atmospheric and Climate Science, ETH, Zurich, Switzerland \\ Correspondence to: Maxime Hervo (maxime.hervo@meteoswiss.ch)
}

Received: 29 January 2016 - Published in Atmos. Meas. Tech. Discuss.: 18 February 2016

Revised: 31 May 2016 - Accepted: 15 June 2016 - Published: 12 July 2016

\begin{abstract}
Imperfections in a lidar's overlap function lead to artefacts in the background, range and overlap-corrected lidar signals. These artefacts can erroneously be interpreted as an aerosol gradient or, in extreme cases, as a cloud base leading to false cloud detection. A correct specification of the overlap function is hence crucial in the use of automatic elastic lidars (ceilometers) for the detection of the planetary boundary layer or of low cloud.

In this study, an algorithm is presented to correct such artefacts. It is based on the assumption of a homogeneous boundary layer and a correct specification of the overlap function down to a minimum range, which must be situated within the boundary layer. The strength of the algorithm lies in a sophisticated quality-check scheme which allows the reliable identification of favourable atmospheric conditions. The algorithm was applied to 2 years of data from a CHM15k ceilometer from the company Lufft. Backscatter signals corrected for background, range and overlap were compared using the overlap function provided by the manufacturer and the one corrected with the presented algorithm. Differences between corrected and uncorrected signals reached up to $45 \%$ in the first $300 \mathrm{~m}$ above ground.

The amplitude of the correction turned out to be temperature dependent and was larger for higher temperatures. A linear model of the correction as a function of the instrument's internal temperature was derived from the experimental data. Case studies and a statistical analysis of the strongest gradient derived from corrected signals reveal that the temperature model is capable of a high-quality correction of overlap artefacts, in particular those due to diurnal variations. The presented correction method has the potential to significantly improve the detection of the boundary layer with gradient-
\end{abstract}

based methods because it removes false candidates and hence simplifies the attribution of the detected gradients to the planetary boundary layer. A particularly significant benefit can be expected for the detection of shallow stable layers typical of night-time situations.

The algorithm is completely automatic and does not require any on-site intervention but requires the definition of an adequate instrument-specific configuration. It is therefore suited for use in large ceilometer networks.

\section{Introduction}

Due to technological advances in recent decades, state-ofthe-art ceilometers can nowadays be considered automatic elastic lidars. They are increasingly used for profiling of aerosols, including the detection of volcanic particles (e.g. Emeis et al., 2011; Flentje et al., 2010; Wiegner et al., 2012) and the determination of the planetary boundary layer (Haeffelin et al., 2012). As for all lidars, there is a zone close to the ground where the telescope field of view does not fully overlap with the laser beam and where geometric and instrumental effects therefore distort the measured backscatter profile. This effect is accounted for with the so-called overlap function, which describes the signal loss due to the overlap effect as a function of altitude. A correct determination of the overlap function is crucial for aerosol profiling in the zone of partial overlap, i.e. in the boundary layer.

The overlap function can theoretically be modelled if the specifications and configuration of the optical elements of the lidar are known (Kuze et al., 1998; Stelmaszczyk et al., 2005). In practice, due to several unknown instrumental ef- 
fects, the precision of such models is generally not sufficient. For example the energy distribution of the laser beam can be ambiguous (Sasano et al., 1979), the transmittance of interference filters may depend on the incident angle (Sasano et al., 1979) or the laser beam might not be well focused on the receiver and will thus alter the measured power (Roberts and Gimmestad, 2002). One of the main issues is the impact of temperature on the optical components (Campbell et al., 2002; Welton and Campbell, 2002).

To determine the overlap function experimentally, several approaches are possible, such as observing a homogeneous atmosphere (Sasano et al., 1979; Welton et al., 2000), using a Raman signal (Wandinger and Ansmann, 2002) or hard target (Vande Hey et al., 2011) or using a reference instrument with a known overlap function (Guerrero-Rascado et al., 2010; Reichardt et al., 2012). Most of these methods require rather costly installations or human intervention and are thus not suited to larger networks of automatic lidars.

The only method that can potentially be applied to a large network at no additional cost is, in our opinion, the use of a vertically homogeneous atmosphere (constant aerosol backscatter and aerosol extinction coefficients). To identify cases with a homogeneous atmosphere, Sasano et al. (1979) proposed to use the ratio between the received power from two altitudes and require that it is stable over time. Since the assumption of a homogeneous atmosphere is not justified across the interface between the boundary layer and the free troposphere, this method is only suited to instruments that reach full overlap within a few hundred metres, i.e. within the boundary layer (Sasano et al., 1979) or for instruments with a correctly specified overlap down to a minimum range within the boundary layer (in this work).

Welton et al. (2000) proposed to perform horizontal measurements such that the assumption of a homogeneous atmosphere also holds for instruments which reach full overlap only after a few thousand metres. Methods using horizontal or inclined measurements are the most common, both in the scientific community and by manufacturers (Campbell et al., 2002; Biavati et al., 2011). However, these methods assume that the overlap function does not change between vertical and inclined alignment of the system, an assumption which may not be justified for certain instruments. Furthermore, the inclination of instruments requires important mechanical developments or human intervention.

Since instrumental parameters are not perfectly constant in time, the overlap function needs to be re-evaluated at regular intervals. Hence, for dense networks of lidars, an automatic approach which requires minimal system modifications is needed. In this study, we propose an extension of the method by Sasano et al. (1979), combined with the assumption that a first guess of the overlap function is available. We will show that this method can be implemented for existing instruments without on-site intervention and that it is suited to large networks of automatic lidars. The algorithm as pre-
Table 1. Instrument parameters.

\begin{tabular}{ll}
\hline Parameter & Value \\
\hline Integration time & $30 \mathrm{~s}$ \\
Bin size & $15 \mathrm{~m}$ \\
Maximum range & $15 \mathrm{~km}$ \\
Overlap-corrected & Yes, TUB120011_ \\
by manufacturer & $20121112 \_1024 . c f g$ \\
Station & Payerne $($ Switzerland, \\
& $\left.6.9417^{\circ} \mathrm{N} ; 46.8117^{\circ} \mathrm{E}\right)$ \\
Altitude & $490 \mathrm{~m}$ \\
Azimuth/zenith angles & $0^{\circ} / 0^{\circ}$ \\
Wavelength & $1064 \mathrm{~nm}$ \\
Average repetition rate & $6.5 \mathrm{KHz}$ \\
Average pulse energy & $8 \mu \mathrm{J}$ \\
Full overlap range & $800 \mathrm{~m}$ \\
\hline
\end{tabular}

sented here is optimized for the CHM15k ceilometer but can in principle be adapted to other instruments.

The paper is organized as follows: the instrument for which the method has been implemented and tested is described in Sect. 2, and in Sect. 3 a detailed description of the method is given. Results are presented in Sect. 4, and in Sect. 5 we discuss temperature effects on the overlap function and propose a model to correct such effects. Examples of the performance of the correction for the determination of the boundary layer height are presented in Sect. 6, followed by a summary and conclusions.

\section{The CHM15k-Nimbus ceilometer}

The CHM15k-Nimbus ceilometer is a biaxial photoncounting lidar $(1064 \mathrm{~nm}, 6.5 \mathrm{KHz}, 8 \mu \mathrm{J})$ manufactured by the company Lufft Mess- und Regeltechnik GmbH (previously manufactured by Jenoptik). The emitter and the receiver are placed next to each other in the optical module, with a centreto-centre distance of $12 \mathrm{~cm}$. More information about a similar instrument can be found in Wiegner and Geiß (2012). For the instrument considered in this study, the lowest level of non-zero (full) overlap is at approximately $180(800) \mathrm{m}$. Its relevant parameters are given in Table 1.

Using a reference instrument, Lufft provides for each optical module an individual overlap function determined in the factory. However, due to mechanical and thermal stress, this overlap function cannot account for changes over time and can thus show significant deficiencies, as shown in Sect. 4.2. It has been noted that artefacts due to differences between the assumed and the true overlap function are visible in the first few hundred metres. Such artefacts are detrimental for various applications, such as the determination of the planetary boundary layer height or the retrieval of aerosol optical properties. 


\section{Method}

\subsection{Physical basis}

The lidar equation relates received power per pulse, $P$, as a function of range, $r$, and time, $t$, to instrumental and atmospheric parameters as follows:

$P(r, t)=$

$\frac{1}{r^{2}} C_{L}(t) C_{\mathrm{CHM}}(t) O(r, t) \beta(r, t) e^{-2 \int_{0}^{r} \alpha\left(r^{\prime}, t\right) \mathrm{d} r^{\prime}}+B(t)$.

$C_{L}$ is the time-dependent calibration factor, and $C_{\mathrm{CHM}}$ is a factor accounting for variations in the sensitivity of the receiver. $C_{\mathrm{CHM}}$ is the product of the variables " $p_{-}$calc" and "scaling" provided by the manufacturer. $\alpha$ and $\beta$ are the extinction and backscatter coefficient, respectively, and $B$ is the background normalized by the number of laser pulses. $O(r t)$ is the range and time-dependent overlap function which can be expressed with a temporally constant overlap function provided by the manufacturer, $O_{\mathrm{CHM}}(r)$, and a correction function, $f_{\mathrm{c}}(r, t)$, as follows:

$O(r, t)=O_{\mathrm{CHM}}(r) / f_{\mathrm{c}}(r, t)$.

The standard instrument output, $\beta_{\text {raw }}$ (variable "beta_raw" provided by the manufacturer), is the normalized and background, range and overlap-corrected signal defined as

$\beta_{\text {raw }}(r, t)=\frac{(P(r, t)-B(t)) r^{2}}{C_{\mathrm{CHM}}(t) O_{\mathrm{CHM}}(r)}$.

We define the corrected instrument output as

$\beta_{\text {corrected }}(r, t)=\beta_{\text {raw }}(r, t) f_{\mathrm{c}}(r, t)$,

which is proportional to the attenuated backscatter coefficient, defined as

$\beta_{\text {att }}(r, t)=\beta(r, t) e^{-2 \int_{0}^{r} \alpha\left(r^{\prime}, t\right) \mathrm{d} r^{\prime}}$.

The factor of proportionality is the calibration factor, as can be shown using Eqs. (1) and (4). The algorithm to calculate the correction function $f_{\mathrm{c}}(r, t)$ is based on two main assumptions:

1. The aerosol extinction and backscatter coefficients are constant in a range interval $[0, R]$ and during the time period of observation (assumption of homogeneous atmosphere).

2. The overlap function is known with low uncertainty in the range interval $\left[R_{\mathrm{OK}}, \infty\right]$, with $R_{\mathrm{OK}} \leq R$.

Under these assumptions, the aerosol lidar ratio (also defined in the literature as extinction-to-backscatter ratio) is constant in the range $[0, R]$. The aerosol backscatter coefficient $\left(\beta_{\mathrm{p}}\right)$ is therefore proportional to the aerosol extinction coefficient $\left(\alpha_{\mathrm{p}}\right)$ in the considered range. The molecular

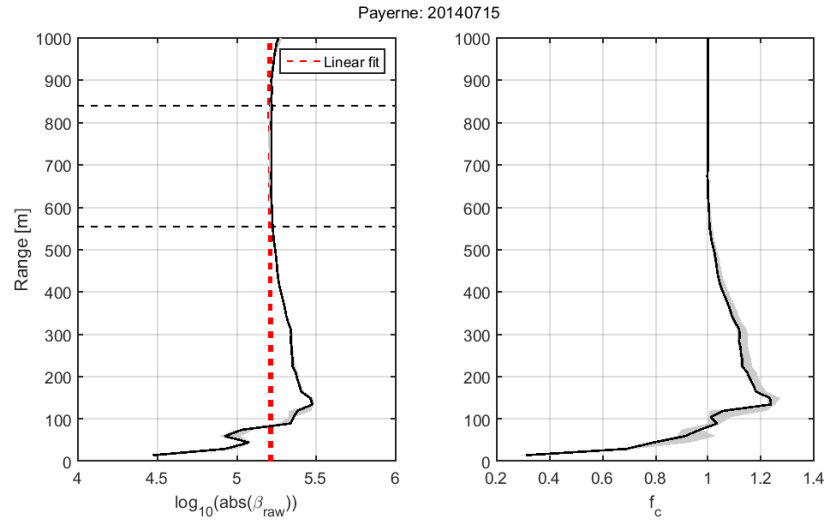

Figure 1. Left panel: logarithm of the absolute value of the range corrected signal measured at Payerne on 15 July 2014 from 00:25 to $01: 20$. The red line represents the linear fit performed between the two black dashed lines. Right panel: corresponding correction function.

backscatter and extinction coefficients, respectively $\beta_{\mathrm{m}}$ and $\alpha_{\mathrm{m}}$, depend on atmospheric density and vary with range.

In the range $[0, R]$ Eqs. (1) to (3) can be written as follows, with time dependence neglected for clarity:

$$
\begin{aligned}
& \log \left(\beta_{\text {raw }}(r)\right)+\log \left(f_{\mathrm{c}}(r)\right)=\log \left(C_{L}\right)+\log \left(\beta_{\mathrm{p}}\right) \\
& -2 \alpha_{\mathrm{p}} r+\log \left(1+\frac{\beta_{\mathrm{m}}(r)}{\beta_{\mathrm{p}}}\right)-2 \int_{0}^{r} \alpha_{\mathrm{m}}\left(r^{\prime}\right) \mathrm{d} r^{\prime} .
\end{aligned}
$$

Using the aerosol lidar ratio $L$ and a molecular lidar ratio equal to $8 \frac{\pi}{3}$, Eq. (6) can be rewritten as follows:

$$
\begin{gathered}
\log \left(\beta_{\text {raw }}(r)\right)+\log \left(f_{\mathrm{c}}(r)\right)=\log \left(C_{L}\right)+\log \left(\frac{\alpha_{\mathrm{p}}}{L}\right) \\
-2 \alpha_{\mathrm{p}} r+\underbrace{\log \left(1+\frac{3 L \alpha_{\mathrm{m}}(r)}{8 \pi \alpha_{\mathrm{p}}}\right)}_{A_{1}(r)} \underbrace{-2 \int_{0}^{r} \alpha_{\mathrm{m}}\left(r^{\prime}\right) \mathrm{D} r^{\prime}}_{A_{2}(r)} .
\end{gathered}
$$

For a standard atmosphere and at a wavelength of $1064 \mathrm{~nm}$, assuming a lidar ratio between 20 and $120 \mathrm{sr}$ and a particle extinction coefficient between 0 and $100 \mathrm{Mm}^{-1}$, the 5th term $\left(A_{2}\right)$ is in the order of $0.01 \%$ of the total signal. $A_{2}$ is neglected for the rest of the calculations. Noting that the 4th term $\left(A_{1}\right)$ is close to straight line, the right hand side of Eq. (7) forms itself, in good approximation, into a straight line:

$\log \left(\beta_{\text {raw }}(r)\right)+\log \left(f_{\mathrm{c}}(r)\right)=A+B r \forall r \in[0, R]$.

Assuming further that $O_{\mathrm{CHM}}(r)$ is correct in the range [ $\left.R_{\mathrm{OK}} R\right]$, i.e. $\log (o(r))=0 \forall r \in\left[R_{\mathrm{OK}} R\right]$, the coefficients $A$ and $B$ are obtained from fitting Eq. (8) to the data in this same range. 

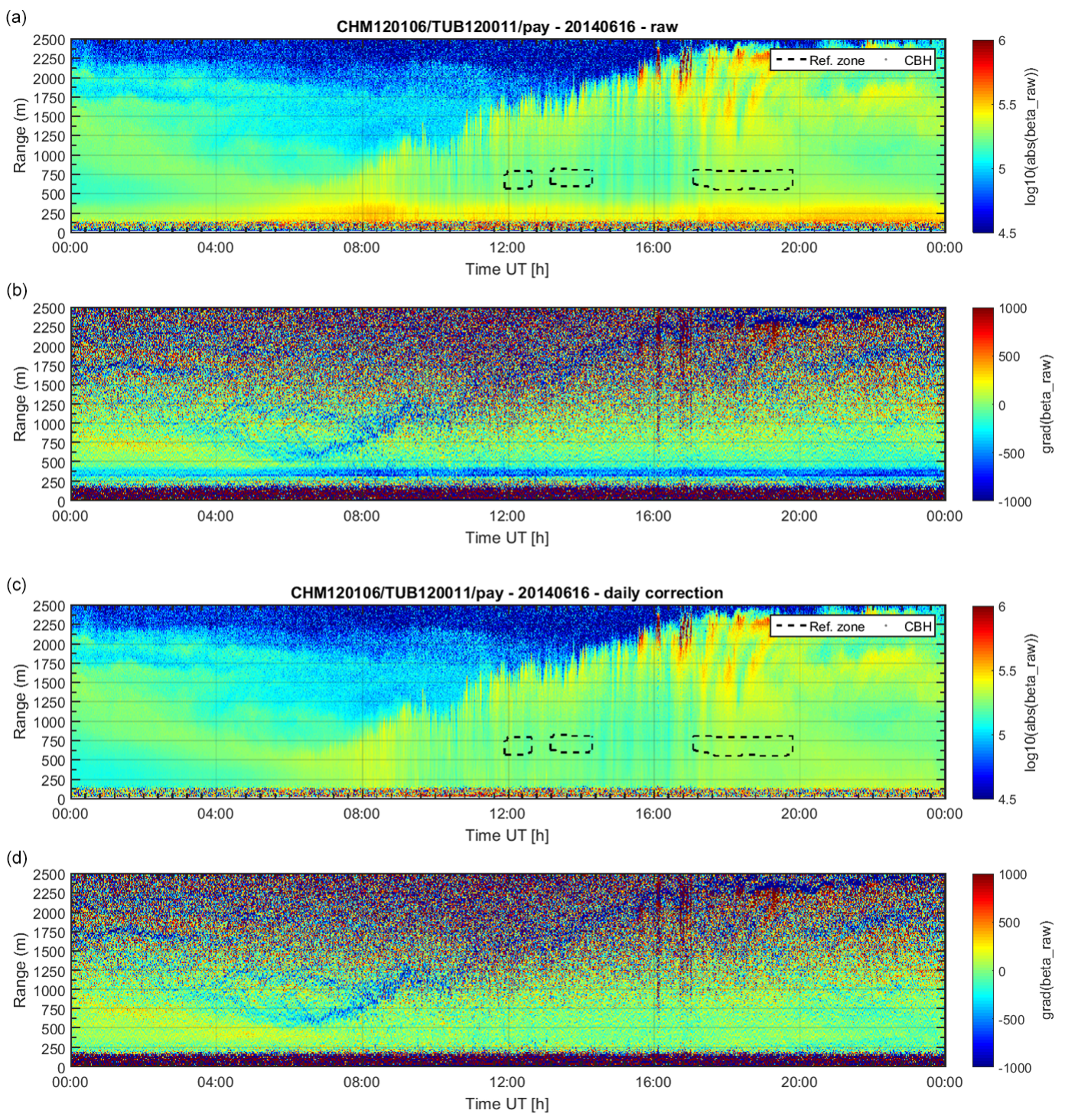

Figure 2. CHM15k measurements at Payerne for 16 June 2014. (a, c): Logarithm of the range corrected signal. (b, d): Gradient of the range corrected signal, (a) and (b): without correction and (c, d): with overlap correction. The reference zones from which the overlap correction was calculated are circled with black dashed lines.

The correction function in the range $[0, R]$ is given by the difference between the fit (right hand side of Eq. 8) and the data as follows:

$f_{\mathrm{c}}(r)=e^{-\left(\log \left(\beta_{\mathrm{raw}}(r)\right)-(A+B r)\right)} \forall r \in[0, R]$.

An example of fitting Eq. (8) to real data is presented on Fig. 1, left panel. The corresponding correction function $f_{\mathrm{c}}$ is represented on the right panel.

\subsection{Outline of the algorithm}

While the approach presented in the previous section is quite straightforward, the implementation of an automatic algorithm is not. The most difficult parts are the selection of favourable atmospheric conditions and the quality control of the result. These two aspects are discussed in detail in Appendix A, while only a brief description of the algorithm is given below.

The algorithm processes a swath of $24 \mathrm{~h}$ of data, for which one overlap correction function is derived. The swath is split into 282 intervals of length $\Delta T=30 \mathrm{~min}$ with starting times $t_{i}$ every $5 \mathrm{~min}$ from 00:00 to 23:30. For each time interval, the mean profile is computed and the fitting interval $\left[R_{\mathrm{OK}} R_{\mathrm{MAX}}\right]$ is determined, where Eq. (8) can be fit to the mean profile. The lower boundary $R_{\mathrm{OK}}$ of the fitting interval represents the lowest range where the overlap function is known with satisfactory accuracy and the upper boundary $R_{\mathrm{MAX}}$ represents the maximum range where the atmosphere is homo- 


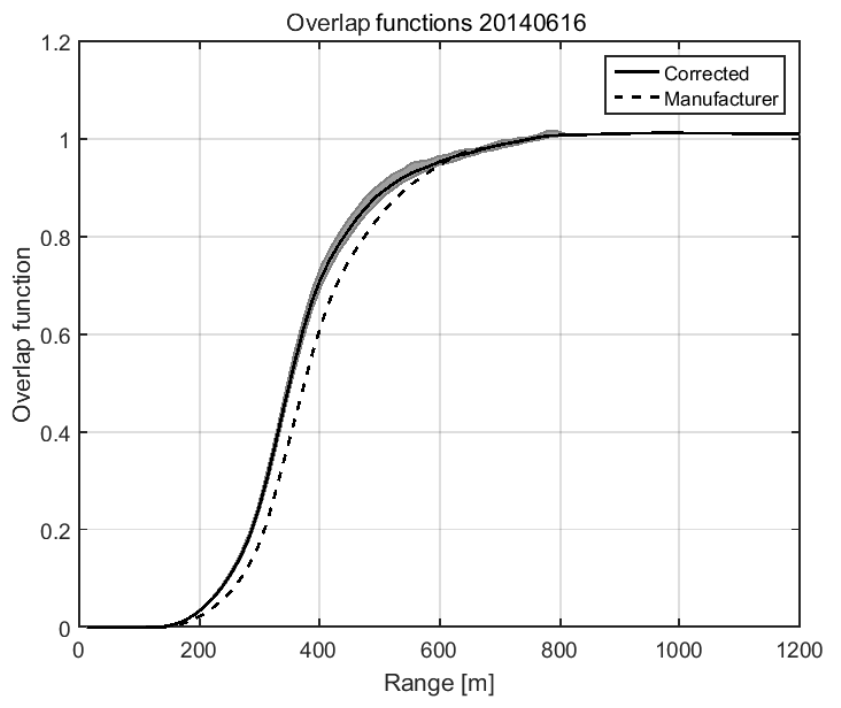

Figure 3. Overlap functions for 16 June 2014. The thick black line is the median overlap function for this day. The dashed line represents the overlap function provided by the manufacturer.

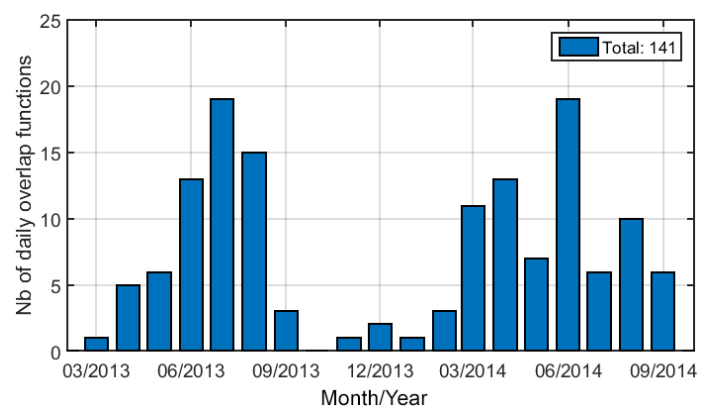

Figure 4. Success rate of the algorithm for 2 years of data.

geneous. Whereas $R_{\mathrm{OK}}$ is instrument specific and constant throughout the processing, $R_{\mathrm{MAX}}$ has to be determined for each time interval (as described in Appendix A). A series of fits is performed in the fitting interval [ $\left.R_{\mathrm{OK}} R_{\mathrm{MAX}}\right]$ from which each one undergoes a sequence of quality checks to evaluate the quality and the plausibility of the fit itself and the obtained overlap correction functions. The final overlap correction function for the entire swath is taken as the median of all overlap correction functions that pass the quality check. This median is hereafter referred to as the "daily correction".

\section{Results}

\subsection{Case study: 16 June 2014}

An example of a successful correction of the overlap function is shown in Fig. 2. This day is representative of a typical planetary boundary layer development (Stull, 1988). The residual layer is visible at night as well as the convective layer that

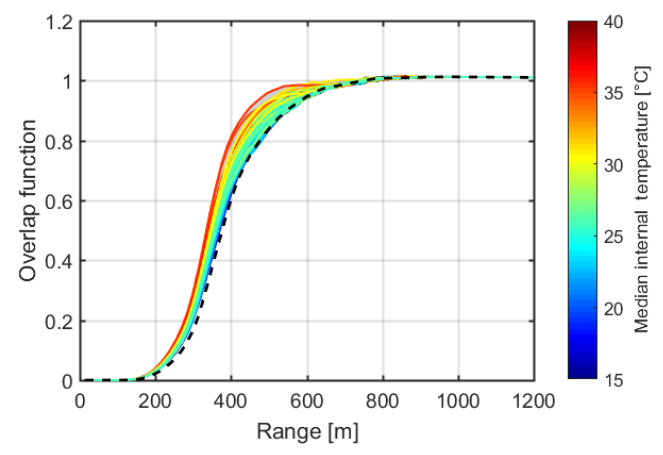

Figure 5. Overlap functions retrieved for Payerne ceilometer in 2013 and 2014. The colours represent the ceilometer internal temperature when the overlap functions were calculated.

developed during the day. An enhancement of the signal centred at $250 \mathrm{~m}$ is visible all day (Fig. 2a). This feature becomes very pronounced when plotting the gradient of the range corrected signal (Fig. 2b) and must be attributed to artefacts induced by inaccuracies in the overlap function provided by the manufacturer.

The algorithm described in Sect. 3.2 was applied for this day. The areas marked with dashed lines indicate the time and height intervals, where Eq. (8) could be fit to the data. For this day, 144 overlap correction functions were selected by the algorithm for 44 out of the 282 time intervals of the swath (for details see Appendix A). The original and the corrected overlap functions are shown in Fig. 3. The overlap function provided by the manufacturer agrees well down to $600 \mathrm{~m}$, which is simply a result of the fact that the function provided by the manufacturer is considered correct down to this altitude. Below, the original overlap function underestimates overlap by up to $45 \%$ around $250 \mathrm{~m}$ (where the overlap value provided by the manufacturer is about 0.2 ).

The median of the corrected overlap functions was applied to the range corrected signal (Fig. 2c) and the gradient recalculated (Fig. 2d). The example demonstrates nicely that the artefact disappears when the overlap correction is applied.

\subsection{Long-term variability}

The algorithm was applied to the ceilometer measurements taken in Payerne from 8 February 2013 to 25 November 2014. The instrument was pointing vertically and achieved a data availability of $99.24 \%$. It was not moved during this time period. Out of the 651 days of operation, an overlap correction could be derived for 141 days $(21.66 \%$ of all the analysed data). The success rate of the algorithm shows a strong seasonal cycle with a higher success rate in summer than in winter (see Fig. 4). This is explained by the fact that in winter, the site is often affected by low cloud and fog. Moreover the homogeneous atmospheric conditions often do not reach the required height due to the shallow boundary layer. 


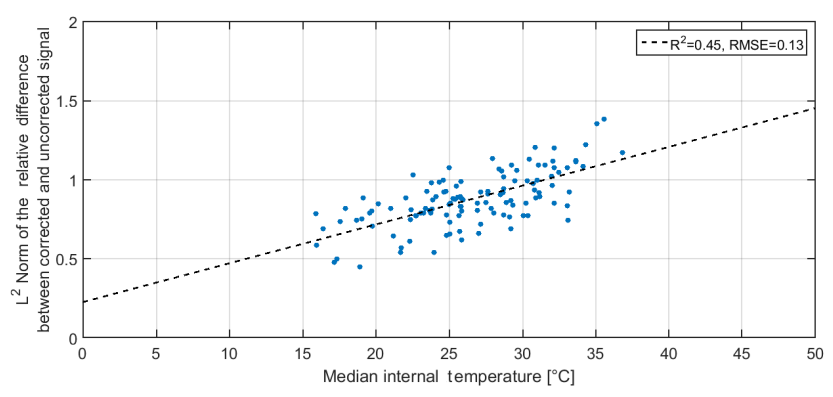

Figure 6. Relative difference between corrected and uncorrected signal against internal temperature.

The obtained overlap functions (Fig. 5) show a large variability and discrepancies up to $50 \%$ with respect to the values provided by the manufacturer. A seasonal cycle is present in the overlap correction with higher values in summer than in winter (not shown).

Assuming that this seasonal cycle is caused by variations in the temperature of the components, the daily overlap functions in Fig. 5 are displayed as a function of the median of the internal temperature measurements corresponding to the successful candidates (see Sect. 3.2 and Appendix A). Figure 5 reveals a clear dependence of the overlap function on the internal temperature with higher values for warmer temperatures. It can further be seen that the overlap function provided by the manufacturer corresponds to corrected overlap functions at low internal temperatures. This temperature dependence is further analysed in the following section and a model to correct for temperature effects is proposed.

\section{Effect of the internal temperature}

Fluctuations of the ambient temperature influence the temperature of the laser and the optical and electronic components. According to the manufacturer, the most temperaturedependent part of the system is the spatial sensitivity of the photodetector (H. Wille, personal communication, 2016). This in turn directly affects the overlap function.

The norm of the relative difference between corrected and uncorrected signal is represented as a function of the internal temperature (Fig. 6) and reveals a clear correlation. The difference between the overlap function provided by the manufacturer and the overlap function calculated by the algorithm increases with the temperature.

The impact of the temperature on the overlap function is now revealed and can be investigated further. Figure 7a shows the relative difference between the corrected and uncorrected signals at each altitude. The shape of the relative difference is in agreement with the artefact described in Sect. 4.1. In this figure, the colour of each line is given by the temperature. The difference between corrected and uncorrected signal reached $45 \%$ at a range of $250 \mathrm{~m}$ for 7 June
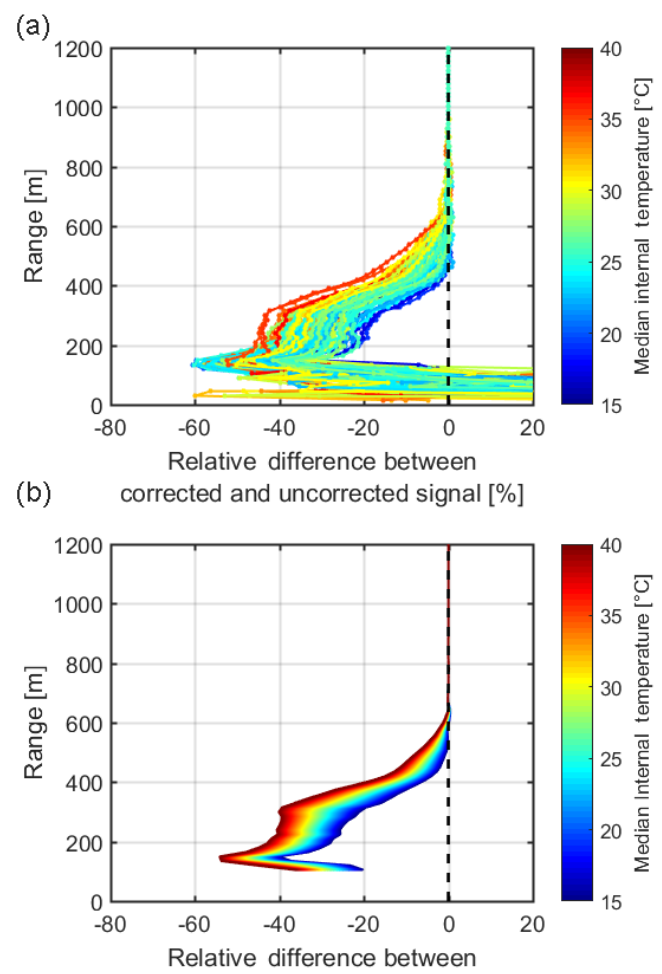

corrected (with model) and uncorrected signal [\%]

Figure 7. Relative difference between corrected and uncorrected signal. Upper panel: from measurements. Lower panel: with model. The colour represents the internal temperature of the instrument.

2014 when the median internal temperature was over $35^{\circ} \mathrm{C}$. On the other hand, when the internal temperature was below $20^{\circ} \mathrm{C}$ on 11 March 2014, the difference decreased to $20 \%$.

In the following, a simple model to correct this temperature effect is described. At each range the relative difference between the corrected and uncorrected signals is assumed to depend linearly on the mean internal temperature. The coefficients for each range are determined by a linear fitting of the relative difference at this range (Fig. 7a). The resulting model is presented in Fig. 7b. To better highlight the temperature dependence in Figs. 5, 6 and 7a, 21 outliers have been identified and discarded (out of the 141 daily overlap function corrections). However to calculate the model coefficients used throughout the study, all data points were considered.

The performance of the model to correct artefacts is assessed in the next section. The major advantages of the model are the possibilities to correct for short-term variations on scales of hours (day/night) and to correct data in real time.

Unfortunately, the coefficients of the temperature model are instrument specific and cannot be used for other instruments or even for other optical modules. However, the algorithm described in Appendix A can be used on any CHM15k to determine the appropriate overlap correction model coefficients if the data set is long enough and covers the entire 

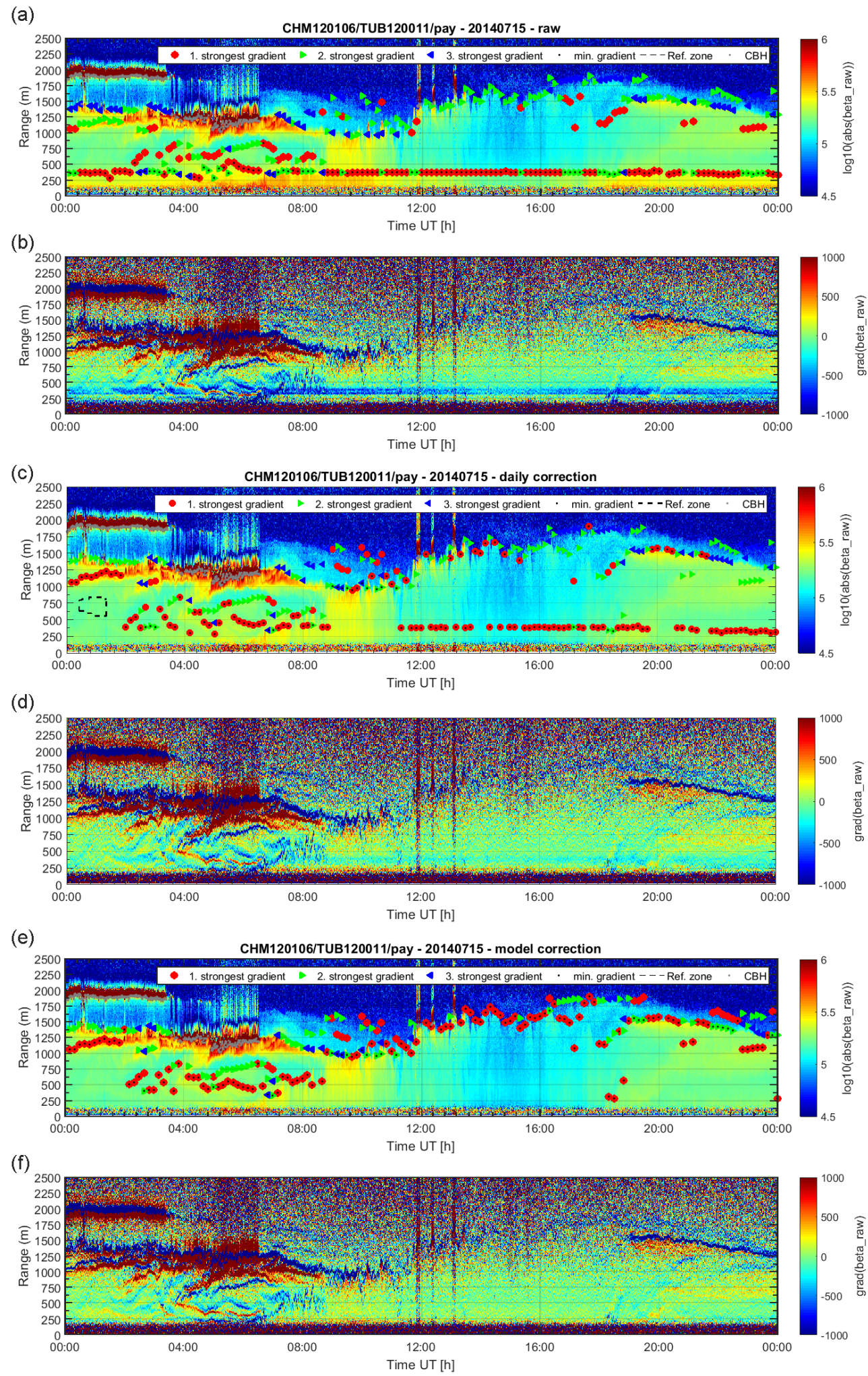

Figure 8. Times series PBL retrievals for 15 July 2014. The red markers show the strongest gradient detected before correction (a, b), with daily correction $(\mathbf{c}, \mathbf{d})$ and with temperature model correction $(\mathbf{e}, \mathbf{f})$. 


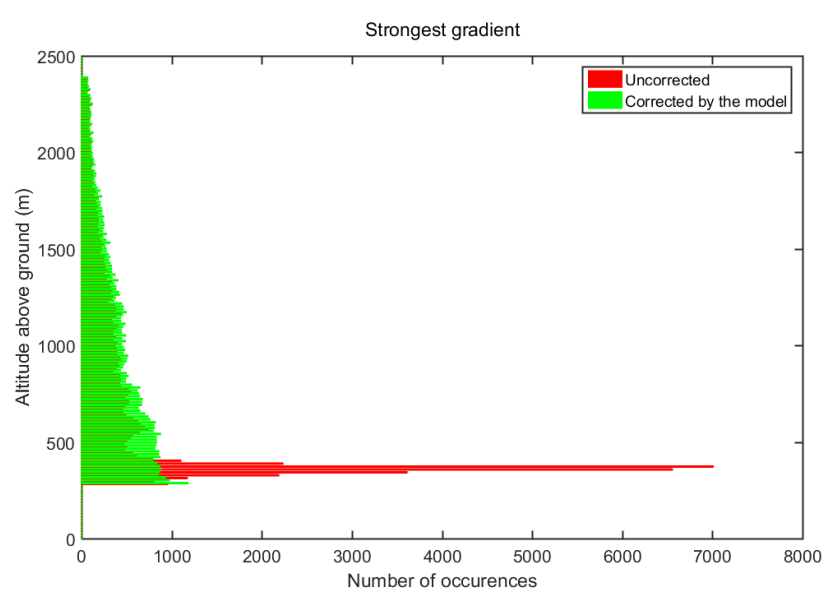

Figure 9. Histogram of the altitude of the strongest 5 min gradients calculated in 2013 and 2014. Uncorrected data are represented in red and data corrected with the temperature model in green.

range of internal temperatures that have to be expected for the site.

\section{Effect of the overlap correction on edge detection}

In almost all boundary layer detection algorithms using aerosols as tracers, the detection of edges or gradients in the backscatter data is the first step. More or less sophisticated approaches are then chosen to attribute one of the detected edges or gradients to the planetary boundary layer height (PBL). This attribution is a very important step in the detection of the PBL but is beyond the scope of this study. This section is therefore limited to demonstrate the effect of our overlap correction method on the detection of aerosol gradients. It is obvious that removing false candidates will also naturally improve the attribution procedure.

\subsection{Case study: 15 July 2014}

In Fig. 8, the performance of the temperature model is compared with corrections made with a single daily overlap function (as in Sect. 4.1). Figure 8a, c and e show the logarithm of the range-corrected signal (called $S$ in Appendix A) measured at Payerne on 15 July 2014. For this day an aerosol layer up to roughly $1500 \mathrm{~m}$ is clearly visible. Figure $8 \mathrm{~b}, \mathrm{~d}$ and $f$ show the corresponding gradient calculated together with the time series of the three strongest gradients as well as the lowest gradient. The gradients were calculated every $5 \mathrm{~min}$ from smoothed range corrected signals (below the cloud base height if any) and gradients of low magnitude were neglected.

If no correction is applied on CHM15k measurements, the strongest gradient is very often located at a constant altitude (Fig. 8a). By applying the algorithm described in the Appendix, an overlap correction was determined using a homo- geneous layer below $800 \mathrm{~m}$ from 00:30 to 01:30 (Figure 8c and d). Using this overlap correction significantly improved the detection of the strongest gradient at the top of the aerosol layer around $1100 \mathrm{~m}$. For this day, the external temperature varied between 11 and $25^{\circ} \mathrm{C}$ and the internal temperature between 22 and $30^{\circ} \mathrm{C}$. This change in temperature had an impact on the overlap function, meaning that the overlap correction retrieved around 01:00 does not perfectly correct the overlap artefact for the entire day. With the temperature model described in Sect. 5, the artefact can be almost perfectly removed for the entire day (Fig. 8f). Consequently, false candidates attributable to the artefact, induced by inaccuracies in the overlap function, could be almost completely removed (Fig. 8e).

\subsection{Long-term variability}

The impact of the overlap correction on the detection of the strongest gradient was tested for the years 2013 and 2014. As in Sect. 6.1, gradients were calculated every $5 \mathrm{~min}$, and the strongest at each time step was selected. The strongest gradient was chosen since this can be considered as a simple attribution solution to the boundary layer (Haeffelin et al., 2012). Figure 9 represents the frequency distribution of the height of this strongest gradient. Uncorrected data are shown in red and the results after the correction with the model in green. For the uncorrected data, a clear spike is visible around $360 \mathrm{~m}$. This spike corresponds to the artefact induced by the uncorrected overlap function described previously. After the correction, this spike disappears and permits more gradient detections between 400 and $1000 \mathrm{~m}$ which are physically meaningful. These gradients were previously masked by some erroneous gradient detections at the altitude of the spike (around $360 \mathrm{~m}$ ).

The presented correction method thus has the potential to significantly improve the detection of the boundary layer using gradient-based methods because it removes false candidates, e.g. in situations of well-mixed convective boundary layer, and hence simplifies the attribution of the detected gradients to the planetary boundary layer. A particularly high benefit can be expected for the detection of shallow stable layers typical in night-time situations.

\section{Summary and conclusions}

Ceilometers are low-cost elastic lidars for unattended operations, and state-of-the-art instruments have the capability to perform aerosol profiling. This opens new applications such as alert systems in case of volcanic ash events, monitoring of long range transport of dust and the determination of the planetary boundary layer height. However, the quality of the range and overlap-corrected signal used in these applications, is often strongly degraded in the first few hundred metres because of imperfections in the specification of the overlap 
function. Here, a method has been presented to correct the overlap function, which is suited for automatic use in large networks, since it does not require any manipulation of the instrument. The method is based on the assumption that the atmosphere is homogeneous over a given time and range interval, in which the overlap function is known to have satisfactory quality. A polynomial of degree one is fit to the data in this interval and a correction function can be computed under the assumption that the atmosphere is also homogeneous from the ground up to the lower boundary of the fitting range interval. The novelty of the method lies in the implementation rather than in the approach itself, the latter being based on Sasano et al. (1979). A series of checks based on the spatio-temporal gradient is performed to identify homogeneous conditions and the appropriate fitting interval. The obtained fits and the derived correction functions for a $24 \mathrm{~h}$ swath of data undergo thorough quality checking using a permutation scheme and stringent tests for the homogeneity of the corrected data.
The analysis of 2 years of data revealed a distinct seasonal cycle in the corrected overlap function. It was demonstrated that these variations are due to variations in the physical temperature of the components. Therefore a model has been developed to compute the corrected overlap function as a function of the internal temperature measured by the instrument, this is the other novel aspect of the presented work. The temperature model has been used to correct data and revealed that gradients related to artefacts induced by the overlap function can be removed to the greatest extent, even during cases where strong temperature differences between day and night are present. The determination of the coefficients the temperature model, the data set used must be representative of a full seasonal cycle, i.e. of at least 1 year. Once the coefficients are determined, the temperature model allows the user to correct ceilometer data in real time and to account for variations on short timescales. It is therefore perfectly suited for application in large networks dedicated to real-time applications. 


\section{Appendix A: Algorithm details}

The different ranges $\left(R_{\ldots} ..\right)$ and thresholds $(\kappa \ldots)$ used in the following paragraphs are explained in Table A1. The values chosen for the implementation of a CHM15k lidar operated in the configuration are specified in Table 1 . The algorithm processes a swath of $24 \mathrm{~h}$ of data for which one overlap correction function is derived. First, the swath is split into 282 intervals of length $\Delta T=30 \mathrm{~min}$, with starting times $t_{i}$ every 5 min from 00:00 to 23:30.

\section{Determination of the fitting intervals}

During this step, it is determined whether during the considered time interval $\left[t_{i}, t_{i}+\Delta T\right], i \in 1 \ldots 282$, there is a range $R_{\text {MAX }}$ below which the atmospheric conditions satisfy the assumptions of homogeneity and thus where fitting intervals $\left[R_{1}, R_{2}\right] \in\left[R_{\mathrm{OK}} R_{\mathrm{MAX}}\right]$ can be constructed and tested.

Note that the $R_{\mathrm{MAX}}$ value may change from one time interval to another and is limited by $R_{\mathrm{MAX}, \mathrm{MAX}}$, usually inside the boundary layer. $R_{\mathrm{MAX}, \mathrm{MAX}}$ determines the maximum range below which homogeneous conditions can be expected. This parameter is not critical for the results but saves computational time, as it restricts the total amount of fitting intervals which need to be tested. $R_{\mathrm{OK}}$ determines the minimum range above which the manufacturer's overlap function is believed to be accurate enough to allow the fitting procedure. The $R_{\mathrm{OK}}$ and $R_{\text {MAX,MAX }}$ values depend on the instrument and site and are fixed for all calculations.

In order to calculate $R_{\mathrm{MAX}}$, the following series of checks are applied:

1. Data availability and bad weather: data availability must be $100 \%$; i.e. the time interval must consist here of 60 non-erroneous profiles, and within the time interval no precipitation or fog (bad weather) should occur, because these events result in saturated, inhomogeneous signals. Weather information is taken here on a profileby-profile basis directly from the ceilometer's output (sky condition index), but it could also be taken from surface station measurements.

2. Cloud and signal-to-noise limitation: the fitting interval should not contain clouds (which result in peaks in the signal) and should not be too noisy. Therefore, the range $R_{\text {CLOUD }}$ of the lowest cloud base height during the whole time interval is identified, as well as the range of the lowest maximum detection height, $R_{\mathrm{SNR}}$. Cloud base heights and maximum detection heights are taken here on a profile-by-profile basis directly from the ceilometer's output, but they could be calculated as well.

3. Test for homogeneity: here we check if characteristic properties of a homogeneous atmosphere are present. The 60 profiles of $\log _{10}\left(\operatorname{abs}\left(\beta_{\text {raw }}\right)\right)$ are considered. For brevity, $\log _{10}\left(\operatorname{abs}\left(\beta_{\text {raw }}\right)\right)$ is hereafter referred to as $S$. At $1064 \mathrm{~nm}$, because of the limited molecular influence, a homogenous atmosphere yields a profile of $S$ close to a line. Therefore, almost vanishing spatial fluctuations of $S$ are expected. These fluctuations can however only be checked starting from the range $R_{\mathrm{OK}}$ where the overlap function is known with satisfactory accuracy. Below this range artificial gradients may appear due to the incorrect manufacturer's overlap correction. Temporal fluctuations in $S$, which should remain small, are checked from the ground up. The ground-level $R_{\mathrm{GROUND}}$ is taken here as the lowest range where the overlap function is larger than 0.05 . Below this range the signal is usually too noisy to be processed. The interval $\left[t_{i}, t_{i}+\Delta T\right]$ is split into subintervals of duration $\Delta T_{\mathrm{s}}=10 \mathrm{~min}$ starting every $30 \mathrm{~s}$ from $t_{i}$ until $t_{i}+\Delta T-\Delta T_{\mathrm{s}}$. All statistical variables and temporal gradients in the following are derived from these subintervals.

\subsection{Temporal homogeneity:}

3.1.1 For each range between $R_{\mathrm{GROUND}}$ and $R_{\mathrm{MAX}, \mathrm{MAX}}$, the ratio of the standard deviation over the median of $S$ is calculated and the maximum value is kept in memory. The lowest range $R_{\mathrm{STD}}$, where this maximum value becomes greater than $\kappa_{1}$, is derived.

3.1.2 For each range between $R_{\text {GROUND }}$ and $R_{\text {MAX,MAX }}$, the norm of the temporal relative gradient

$$
\nabla_{X}^{*} S=\frac{\left|\nabla_{X} S\right|}{|S|}
$$

is calculated, with $\nabla_{X}$ being calculated with a Sobel operator (convolution-based edge detector). The lowest range $R_{\text {GRADX }}$ where

$$
\begin{aligned}
& \max \left(\nabla_{X}^{*} S(r, t)\right) \geq \kappa_{2} \\
& \text { with } \quad(r, t) \in\left[R_{\mathrm{GROUND}}, R_{\mathrm{GRADX}}\right] \times\left[t_{i}, t_{i}+\right. \\
& \Delta T], \text { is derived. }
\end{aligned}
$$

3.2 Spatial homogeneity: for each range between $R_{\mathrm{OK}}$ and $R_{\mathrm{MAX}, \mathrm{MAX}}$, the norm of the spatial relative gradient

$\nabla_{Y}^{*} S=\frac{\left|\nabla_{Y} S\right|}{|S|}$

is calculated, with $\nabla_{Y}$ being calculated with a Sobel operator. The lowest range $R_{\mathrm{GRADY}}$ where

$\max \left(\nabla_{Y}^{*} S(r, t)\right) \geq \kappa_{2}$

with $(r, t) \in\left[R_{\mathrm{OK}}, R_{\mathrm{GRADY}}\right] \times\left[t_{i}, t_{i}+\Delta T\right]$ is derived. 
Table A1. Algorithm parameters.

\begin{tabular}{|c|c|c|}
\hline Parameter & Description & Value \\
\hline$R_{\text {GROUND }}$ & Lowest measurement range & $\begin{array}{l}\text { Lowest range where the overlap function } \\
\text { provided by the manufacturer } \geq 5 \%\end{array}$ \\
\hline$R_{\mathrm{OK}}$ & $\begin{array}{l}\text { Range above which the manufacturer's overlap function is believed to } \\
\text { be accurate }\end{array}$ & $\begin{array}{l}\text { Lowest range where the overlap function } \\
\text { by the manufacturer } \geq 80 \%\end{array}$ \\
\hline$R_{\text {MAX,MAX }}$ & Highest allowed range for the fitting & $1200 \mathrm{~m}$ \\
\hline$R_{O_{\mathrm{CHM}}=1}$ & $\begin{array}{l}\text { Lowest range where the manufacturer's overlap function reaches } 1 \\
\text { (full overlap) }\end{array}$ & $\begin{array}{l}\text { Lowest range where the overlap function } \\
\text { provided by the manufacturer } \geq 100 \%\end{array}$ \\
\hline$\Delta R_{\mathrm{MIN}}$ & Minimum length of the fitting intervals & $150 \mathrm{~m}$ \\
\hline$\kappa_{1}$ & Upper threshold for the ratio of the standard deviation over the median & 0.01 \\
\hline$\kappa_{2}$ & Upper threshold for the relative gradient & 0.05 \\
\hline$\kappa_{3}$ & Upper threshold for the mean relative gradient & 0.015 \\
\hline$\kappa_{4}$ & Lower threshold for the slope of the linear fit & $\frac{-2}{\log (10)} 10^{-5}$ \\
\hline$\kappa_{5}$ & Upper threshold for the slope of the linear fit & $\frac{-2}{\log (10)} 10^{-7}$ \\
\hline$\kappa_{6}$ & Lower threshold for the $y$ axis offset of the linear fit & 4.75 \\
\hline$\kappa_{7}$ & Upper threshold for the $y$ axis offset of the linear fit & 6 \\
\hline$\kappa_{8}$ & Upper threshold for the relative RMSE of the linear fit & 0.0005 \\
\hline$\kappa 9$ & $\begin{array}{l}\text { Upper threshold for the ratio between the maximum values of the corrected } \\
\text { overlap function and the manufacturer's overlap function }\end{array}$ & 1.01 \\
\hline$\kappa_{10}$ & $\begin{array}{l}\text { Upper threshold for the relative error of the corrected overlap function w.r.t. } \\
\text { the manufacturer's overlap function in the full overlap region }\end{array}$ & 0.01 \\
\hline$\kappa_{11}$ & Lower threshold for the slope of the corrected overlap function & -0.00025 \\
\hline
\end{tabular}

3.3 Spatial and temporal homogeneity: for each range between $R_{\mathrm{OK}}$ and $R_{\mathrm{MAX} \text { MAX }}$ the norm of the two dimensional relative gradient is calculated with the following equation:

$$
\nabla_{X Y}^{*} S=\sqrt{\left|\frac{\nabla_{X} S}{S}\right|^{2}+\left|\frac{\nabla_{Y} S}{S}\right|^{2}} .
$$

The lowest range $R_{\mathrm{GRADXY}}$ is derived, where

$$
\max \left(\nabla_{X Y}^{*} S(r, t)\right) \geq \kappa_{2}
$$

or where

$$
\begin{aligned}
& \operatorname{mean}\left(\nabla_{X Y}^{*} S(r, t)\right) \geq \kappa_{3}, \\
& \text { with }(r, t) \in\left[R_{\mathrm{OK}}, R_{\mathrm{GRADXY}}\right] \times\left[t_{i}, t_{i}+\Delta T\right] .
\end{aligned}
$$

Once these bad weather, cloud, noise and homogeneity tests are completed, the upper boundary of the fitting interval is set to

$R_{\text {MAX }}=$

$\min \left(R_{\mathrm{CLOUD}} R_{\mathrm{SNR}} R_{\mathrm{STD}} R_{\mathrm{GRADX}} R_{\mathrm{GRADY}} R_{\mathrm{GRADXY}}\right)$.

If $R_{\mathrm{MAX}}$ is smaller than $R_{\mathrm{OK}}+\Delta R_{\mathrm{MIN}}$ the time interval $\left[t_{i}, t_{i}+\Delta T\right]$ is rejected. If $R_{\mathrm{MAX}}>R_{\mathrm{MAX}, \mathrm{MAX}}$, we set its value to $R_{\text {MAX,MAX, because the fitting part and subsequent }}$ quality check in the following are computationally costly.

\section{Quality check of the fits and determination of a set of overlap correction candidates}

The range interval [ $R_{\mathrm{OK}} R_{\mathrm{MAX}}$ ] is now split into all possible intervals $\left[R_{1}, R_{2}\right]$ on the discrete range grid and of length equal to or larger than $\Delta R_{\mathrm{MIN}}$ that fit into $\left[R_{\mathrm{OK}} R_{\mathrm{MAX}}\right]$. In each such range interval $\left[R_{1}, R_{2}\right]$ the mean profile of $S$ for the time interval $\left[t_{i}, t_{i}+\Delta T\right]$ is fit with a straight line according to Eq. (8) and the obtained linear fits undergo the following series of checks:

4. Plausibility of slope and ground value: under homogeneous conditions, the slope of the fit is approxi- 
mately $\frac{-2}{\log (10)} \alpha_{\mathrm{p}}$ and the $y$ axis offset is approximately $\log _{10}\left(C_{L}\right)+\log _{10}\left(\frac{\alpha_{\mathrm{p}}}{L}\right)$. Note that the factor $\log (10)$ is needed because $S$ is calculated with the $\log$ with base 10. Bounds based on estimations of reasonable values for $\alpha_{\mathrm{p}}, C_{L}$ and $L$ can be set such that the slope must lie between $\kappa_{4}$ and $\kappa_{5}$ and the $y$ axis offset must lie between $\kappa_{6}$ and $\kappa_{7}$.

5. Goodness of fit: the RMSE of the fit divided by its mean must be smaller than $\kappa_{8}$.

The linear fits that successfully passed these checks form a set of candidates to be used to derive the overlap correction.

\section{Quality check of the overlap correction candidates}

For each such candidate, with its fitting range $\left[R_{1} R_{2}\right]$ as unique identifier, the corrected overlap function, $O_{\text {corr }}$, is computed using Eqs. (2) and (9) where $O_{\operatorname{corr}\left(R \geq R_{2}\right)}=$ $O_{\mathrm{CHM}}\left(R \geq R_{2}\right)$. The corrected overlap function is checked for plausibility with the following series of checks:

6. Maximum value: corrected overlap functions showing unphysically high values are discarded. Therefore, $\max \left(O_{\text {corr }}\right) / \max \left(O_{\mathrm{CHM}}\right)$ must be smaller than $\kappa_{9}=$ 1.01 .

7. Small relative error with respect to the manufacturer's overlap in the full overlap region: the relative error $\frac{\left|O_{\text {corr }}(R)-O_{\mathrm{CHM}}(R)\right|}{\left|O_{\mathrm{CHM}}(R)\right|}$ must be smaller than $\kappa_{10}=0.01$ for the ranges $R \geq R_{O_{\mathrm{CHM}}=1}$ (range of full overlap, where it is assumed that the manufacturer's overlap is exact). For the CHM15k, $R_{O_{\mathrm{CHM}}=1}$ can vary from instrument to instrument between 500 and $2000 \mathrm{~m}$.

8. Temporal and spatial homogeneity: the 60 profiles of $S_{\text {corr }}=\log _{10}\left(\operatorname{abs}\left(\beta_{\text {raw }_{\text {corrected }}}\right)\right)$ obtained from Eq. (3) with the corrected overlap function (Eq. 2) are now considered. The relative spatio-temporal gradients $\nabla_{X Y}^{*} S_{\text {corr }}$ are calculated as in test 3.3 "Spatial and temporal homogeneity". Temporal and spatial fluctuations are expected to be small for all ranges from $R_{\mathrm{GROUND}}$ to $R_{2}$. Therefore the following conditions must be satisfied:

$\max \left(\nabla_{X Y}^{*} S_{\text {corr }}(r, t)\right)<\kappa_{2}$

$\left.\operatorname{mean}\left(\nabla_{X Y}^{*} S_{\text {corr }}(r, t)\right)<\kappa_{3}\right)$

with $(r, t) \in\left[R_{\mathrm{GROUND}}, R_{2}\right] \times\left[t_{i}, t_{i}+\Delta T\right]$.
9. Monotonic increase: an overlap function should increase monotonically up to the range of full overlap. Therefore only a small negative slope (resulting from limited inhomogeneities in the correction) should be allowed. The slope of $O_{\text {corr }}$, computed with a Savitzky-Golay filter (Savitzky and Golay, 1964) of width 5 and order 3 , must be larger than $\kappa_{11}=$ $-0.00025 \mathrm{~m}^{-1}$ between 0 and $R_{2}$, i.e. a decrease of maximum $0.015 \% \mathrm{~m}^{-1}$ is allowed.

\section{Final selection}

All successful candidates obtained from each time interval $\left[t_{i}, t_{i}+\Delta T\right]$ are kept in a global list for the entire swath $(24 \mathrm{~h})$. For the entire swath a minimum of 15 candidates must be obtained, otherwise the swath is rejected for the calculation of an overlap correction. To ensure that the overlap function does not change much within one swath, each candidate is checked in the time interval of all other candidates, with test 8 and test 3.1.1 from range $R_{\mathrm{GROUND}}$ to their ranges $R_{2}$. From the successful candidates, outliers are removed (an outlier lies outside three interquartile ranges from the median with respect to both slope and $y$ axis offset). If the final set contains more than 10 candidates, the final overlap correction is the median overlap correction. Otherwise, the swath is rejected. 
Acknowledgements. This study has been financially supported by ICOS-CH and E-PROFILE (EUMETNET). The authors would further like to thank Gianni Martucci, Robert J. Sica, Martial Haeffelin and Barbara Althaus for their constructive remarks. The authors would like to acknowledge the contribution of the COST Action ES1303 (TOPROF). The authors are grateful to Kornelia Pönitz and Holger Wille (Lufft) for technical information about the CHM15k.

Edited by: U. Wandinger

\section{References}

Biavati, G., Donfrancesco, G. D., Cairo, F., and Feist, D. G.: Correction scheme for close-range lidar returns, Appl. Opt., 50, 5872, doi:10.1364/AO.50.005872, 2011.

Campbell, J. R., Hlavka, D. L., Welton, E. J., Flynn, C. J., Turner, D. D., Spinhirne, J. D., Scott, V. S., and Hwang, I. H.: Full-Time, Eye-Safe Cloud and Aerosol Lidar Observation at Atmospheric Radiation Measurement Program Sites: Instruments and Data Processing, J. Atmospheric Ocean. Technol., 19, 431-442, doi:10.1175/15200426(2002)019<0431:FTESCA>2.0.CO;2, 2002.

Emeis, S., Forkel, R., Junkermann, W., Schäfer, K., Flentje, H., Gilge, S., Fricke, W., Wiegner, M., Freudenthaler, V., Groß, S., Ries, L., Meinhardt, F., Birmili, W., Münkel, C., Obleitner, F., and Suppan, P.: Measurement and simulation of the 16/17 April 2010 Eyjafjallajökull volcanic ash layer dispersion in the northern Alpine region, Atmos. Chem. Phys., 11, 2689-2701, doi:10.5194/acp-11-2689-2011, 2011.

Flentje, H., Claude, H., Elste, T., Gilge, S., Köhler, U., PlassDülmer, C., Steinbrecht, W., Thomas, W., Werner, A., and Fricke, W.: The Eyjafjallajökull eruption in April 2010 - detection of volcanic plume using in-situ measurements, ozone sondes and lidar-ceilometer profiles, Atmos. Chem. Phys., 10, 10085-10092, doi:10.5194/acp-10-10085-2010, 2010.

Guerrero-Rascado, J. L., Costa, M. J., Bortoli, D., Silva, A. M., Lyamani, H., and Alados-Arboledas, L.: Infrared lidar overlap function: an experimental determination, Opt. Express, 18, 20350, doi:10.1364/OE.18.020350, 2010.

Haeffelin, M., Angelini, F., Morille, Y., Martucci, G., Frey, S., Gobbi, G. P., Lolli, S., O’Dowd, C. D., Sauvage, L., XuerefRémy, I., Wastine, B., and Feist, D. G.: Evaluation of MixingHeight Retrievals from Automatic Profiling Lidars and Ceilometers in View of Future Integrated Networks in Europe, Bound.Lay, Meteorol., 143, 49-75, doi:10.1007/s10546-011-9643-z, 2012.

Kuze, H., Kinjo, H., Sakurada, Y., and Takeuchi, N.: Fieldof-View Dependence of Lidar Signals by Use of Newtonian and Cassegrainian Telescopes, Appl. Opt., 37, 3128-3132, doi:10.1364/AO.37.003128, 1998.
Reichardt, J., Wandinger, U., Klein, V., Mattis, I., Hilber, B., and Begbie, R.: RAMSES: German Meteorological Service autonomous Raman lidar for water vapor, temperature, aerosol, and cloud measurements, Appl. Opt., 51, 8111, doi:10.1364/AO.51.008111, 2012.

Roberts, D. W. and Gimmestad, G. G.: Optimizing lidar dynamic range by engineering the crossover region, Proc. SPIE 4723, Laser Radar Technology and Applications VII, 120, 2002.

Sasano, Y., Shimizu, H., Takeuchi, N., and Okuda, M.: Geometrical form factor in the laser radar equation: an experimental determination, Appl. Opt., 18, 3908, doi:10.1364/AO.18.003908, 1979.

Savitzky, A. and Golay, M. J. E.: Smoothing and Differentiation of Data by Simplified Least Squares Procedures, Anal. Chem., 36, 1627-1639, doi:10.1021/ac60214a047, 1964.

Stelmaszczyk, K., Dell'Aglio, M., Chudzyński, S., Stacewicz, T., and Wöste, L.: Analytical function for lidar geometrical compression form-factor calculations, Appl. Opt., 44, 1323, doi:10.1364/AO.44.001323, 2005.

Stull, R. B.: An Introduction to Boundary Layer Meteorology, Springer Science \& Business Media, 1988.

Vande Hey, J., Coupland, J., Foo, M. H., Richards, J., and Sandford, A.: Determination of overlap in lidar systems, Appl. Opt., 50, 5791, doi:10.1364/AO.50.005791, 2011.

Wandinger, U. and Ansmann, A.: Experimental Determination of the Lidar Overlap Profile with Raman Lidar, Appl. Opt., 41, 511514, doi:10.1364/AO.41.000511, 2002.

Welton, E. J. and Campbell, J. R.: Micropulse Lidar Signals: Uncertainty Analysis, J. Atmospheric Ocean. Technol., 19, 2089-2094, doi:10.1175/1520-0426(2002)019<2089:MLSUA>2.0.CO;2, 2002.

Welton, E. J., Voss, K. J., Gordon, H. R., Maring, H., Smirnov, A., Holben, B., Schmid, B., Livingston, J. M., Russell, P. B., Durkee, P. A., Formenti, P., and Andreae, M. O.: Ground-based lidar measurements of aerosols during ACE-2: instrument description, results, and comparisons with other ground-based and airborne measurements, Tellus B, 52, 636-651, doi:10.1034/j.16000889.2000.00025.x, 2000.

Wiegner, M. and Geiß, A.: Aerosol profiling with the Jenoptik ceilometer CHM15kx, Atmos. Meas. Tech., 5, 1953-1964, doi:10.5194/amt-5-1953-2012, 2012.

Wiegner, M., Gasteiger, J., Groß, S., Schnell, F., Freudenthaler, V., and Forkel, R.: Characterization of the Eyjafjallajökull ashplume: Potential of lidar remote sensing, Phys. Chem. Earth, 4546, 79-86, doi:10.1016/j.pce.2011.01.006, 2012. 